\title{
REDUCED BASIS METHOD FOR OPTIMAL CONTROL OF UNSTEADY VISCOUS FLOWS
}

\author{
K. ITO $^{\dagger}$ AND S. S. RAVINDRAN ${ }^{\dagger} \ddagger$ \\ NORTH CAROLINA STATE UNIVERSITY, RALEIGH, NORTH CAROLINA 27695
}

\begin{abstract}
In this article we discuss the reduced basis method (RBM) for optimal control of unsteady viscous flows. RBM is a reduction method in which one can achieve the versatility of the finite element method or another for that matter and gain significant reduction in the number of degrees of freedom. The essential idea in this method is to define a reduced order subspace spanned by few basis elements and then obtain the solution via a Galerkin projection. We present several ways to define this subspace. Feasibility of the approach is demonstrated on two boundary control problems in cavity and wall bounded channel flows. Control action is effected through boundary surface movement on part of the solid wall. Application of RBM to the control problems leads to finite dimensional optimal control problems which are solved using Newton's method. Through computational experiments we demonstrate the feasibility and applicability of the reduced basis method for control of unsteady viscous flows.
\end{abstract}

\section{Introduction}

Active control of fluid dynamical system for the design of advanced fluid dynamical technology is becoming an increasingly important tool. There is an extensive literature devoted to this actively growing field both by theoretical and experimental approaches; see $[9,5,7,22,20,23,11,8,13,2,3,19,14]$. The computational feasibility of some of these approaches were demonstrated in $[9,10,11]$. However, they involve computationally intensive algorithms which are prohibitively expensive for more complex real-world problems. The goal of this paper is to provide a feasible computational method based on reduced order models (ROM) for the active control problems arising in nonlinear fluid dynamic systems.

We construct the reduced order models using the so-called reduced basis method (RBM). The RBM appears to have been first proposed in [1] and [15] which was developed further in [16] in the context of structural mechanics problems. The first use of RBM for optimal control problems in fluid flows can be found in [12] where steady state control problems in viscous flows were discussed.

In this article, we will focus on implementation of reduced basis method for optimal control problems in unsteady viscous flows. The reduced basis method uses basis functions which are generated from the problem that is being solved. This is in contrast to the traditional numerical methods such as finite difference method which uses grid functions as basis functions or finite elements method which uses piecewise polynomials for this purpose. There are several approaches available for the generation of reduced basis functions. We will discuss three such approaches, namely, the Taylor approach, the Lagrange approach and the Hermite approach. The Taylor approach uses solutions at a reference point in the parameter space along with their derivatives with respect to the parameter as basis functions. In the Lagrange approach one uses solutions of the problem at various parameter values as basis functions. Finally the Hermite approach is a hybrid of Lagrange and Taylor approaches which uses solu-

\footnotetext{
$\ddagger$ Currently an NRC Fellow at Flow Modeling and Control Branch, NASA Langley Research Center, Mail Stop 170, Hampton, VA 23681 (s.s.ravindran@larc.nasa.gov);

http://fmcb.larc.nasa.gov/ ravi.

${ }^{\dagger}$ Center for Research in Scientific Computing, Department of Mathematics, North Carolina State University, Raleigh, NC 27695-8205 (kito@eos.ncsu.edu)
} 
tions and their first derivatives of the problem at various parameter values as basis functions.

We demonstrate the advantages of the RBM approach for unsteady optimal control problems by implementing it on two control problems: (i) the control of driven cavity flow and (ii) the control of wall-bounded channel flow. A number of works have been devoted to control of cavity and channel flows. Theoretical and numerical works on control of cavity flows are discussed in [5], [4], [10], [11] and [12] using various control mechanisms. The control of channel flows are reported in [13], [8], [2], [3], [19], [10], [11] and [12]. In [13], [3], [19] and [8] experimental results are presented, and a related open-loop control simulations is given [2]. In [10], [11] and [12] computational works using optimal control techniques are presented.

The plan of the paper is as follows. In the remainder of this section we establish some notations that will be used throughout the paper. In $\S I I$, we will present RBM and the construction of reduced order model in an abstract setting. We will also discuss a number of ways to construct reduced basis elements. In §III, we will illustrate the construction of reduced order model for a model problem in unsteady viscous flows and use a channel flow problem to demonstrate the effectiveness of ROM in capturing the essential physics. In $\S I V$, we will show the application of reduced basis method for optimal control problems in viscous incompressible flows. Construction of reduced order control problem is shown and a numerical method to solve such reduced order control problems is then presented. In $\S \mathrm{V}$, computational results are presented for control of driven cavity and channel flows.

\section{A. Notation}

We denote by $L^{2}(\Omega)$ the collection of square-integrable functions defined on flow region $\Omega \subset \mathbb{R}^{2}$ and we denote the associated norm by $\|\cdot\|_{0}$. Let

$$
H^{1}(\Omega)=\left\{v \in L^{2}(\Omega): \frac{\partial v}{\partial x_{i}} \in L^{2}(\Omega), \quad i=1,2\right\}
$$

and the norm on it be $\|\cdot\|_{1}$. We denote by $L^{2}\left(0, T ; H^{1}\right)$ the space of all measurable functions $f:(0, T) \rightarrow H^{1}$ such that $\|f\|_{L^{2}\left(0, T ; H^{1}\right)}=\left(\int_{0}^{T}\|f\|_{1}^{2} d t\right)^{\frac{1}{2}}<\infty$. Vectorvalued counterparts of these spaces are denoted by bold-face symbols, e.g., $\mathbf{H}^{1}(\Omega)=$ $\left[H^{1}(\Omega)\right]^{2}$. The $L^{2}(\Omega)$ or $\mathbf{L}^{2}(\Omega)$ inner product is denoted by $(\cdot, \cdot)$. We denote the inner products for $L^{2}(\Gamma)$ or $\mathbf{L}^{2}(\Gamma)$ by $(\cdot, \cdot)_{\Gamma}$, where $\Gamma$ denotes the boundary of $\Omega$.

\section{The Reduced Basis Method and Reduced Basis Subspace}

\section{A. The Reduced Basis Subspace}

In order to illustrate the reduced basis subspace, we first assume that we are dealing with nonlinear dynamics about stable equilibrium points. Consider the parameterized stationary problem

$$
E(\mathbf{y}, \mu)=0 \quad \text { for } \mu \in \Lambda, \mathbf{y} \in \mathbf{X},
$$

where $\mathbf{X}$ is a finite dimensional space and $\mu \in \Lambda$ represents some physical parameter, for example, Reynolds number, Mach number, time variable t for evolution equation or control variables. If finite element were used to approximate this problem, $\mathbf{X}$ would be a piecewise polynomial space. However, the choice for reduced basis subspace is 
different. We construct its elements based on the interpolation of solution function $\mathbf{y}(\mu)$ as follows.

The Taylor Subspace. In this choice, one uses the Taylor expansion of function $\mathbf{y}(\mu)$ at a reference value of $\mu$, say $\mu^{*}$ and the reduced basis subspace $\mathbf{X}_{r}$ is defined as

$$
\mathbf{X}_{r}=\operatorname{span}\left\{\mathbf{y}_{j}=\left.\frac{\partial^{j} \mathbf{y}}{\partial \mu^{3}}\right|_{\mu=\mu^{*}}, \quad j=1, \ldots \ldots, N_{r}\right\}
$$

The $j$-th derivative $\mathbf{y}_{j}$ can be calculated from the equations resulting from successive differentiation of (1), i.e.

$$
E_{\mathbf{y}}\left(\mathbf{y}_{0}, \mu_{0}\right) \mathbf{y}_{j}=F_{j}\left(\mathbf{y}_{0}, \mathbf{y}_{1}, \ldots, \mathbf{y}_{j-1}, \mu^{*}\right) .
$$

For example, $\mathbf{y}_{1}$ satisfies the equation

$$
E_{\mathbf{y}}\left(\mathbf{y}_{0}, \mu^{*}\right) \mathbf{y}_{1}=-E_{\mu}\left(\mathbf{y}_{0}, \mu^{*}\right) .
$$

We note here that each $\mathbf{y}_{j}$ can be obtained from its predecessors by solving a linear system with the same linear operator $E_{\mathbf{y}}\left(\mathbf{y}_{0}, \mu^{*}\right)$.

The Lagrange Subspace. In this case, the basis elements are solutions of the nonlinear problem under study at various parameter values $\mu_{j}$. The reduced basis subspace is given by

$$
\mathbf{X}_{r}=\operatorname{span}\left\{\mathbf{y}^{j} \mid \mathbf{y}^{j}=\mathbf{y}\left(\mu_{j}\right), \quad j=1, \ldots ., N_{r}\right\} .
$$

A possible advantage in this choice is that updating the basis elements can be done one basis element at a time instead of generating the whole space.

The Hermite Subspace. This is a hybrid of the Lagrange and Taylor approach. The basis elements are solutions and their first derivatives at various parameter values $\mu_{j}$. The reduced basis subspace is given by

$$
\mathbf{X}_{r}=\operatorname{span}\left\{\mathbf{y}^{j}=\mathbf{y}\left(\mu_{j}\right) \quad \text { and }\left.\frac{\partial \mathbf{y}}{\partial \mu}\right|_{\mu=\mu_{j}}, \quad j=1, \ldots \ldots, \widetilde{N_{r}}\right\}
$$

\section{B. The Reduced Order Model}

Let us now turn to the definition of reduced order model. Suppose we have a reduced basis space $\mathbf{X}_{r}$ with a basis $\left\{\boldsymbol{\Phi}_{i}\right\}_{i=1}^{N_{r}}$. Then we project the equation (1) by the Galerkin approximation to obtain the reduced oder model: For $\mathbf{y}^{r}=\sum_{i=1}^{N_{r}} \alpha_{i} \boldsymbol{\Phi}_{i}$

$$
E_{r}\left(\mathbf{y}^{r}, \mu\right)_{i}=\left(E\left(\mathbf{y}^{r}, \mu\right), \Phi_{i}\right), \quad \mathrm{i}=1, \ldots \ldots, N_{r} .
$$

We next turn to control dynamic equations which is the focus of this study. For control dynamic equation

$$
\frac{d \mathbf{y}}{d t}+E(\mathbf{y}, U)=0,
$$

where $U$ is the control variable, one can generate reduced basis elements $\left\{\mathbf{y}_{i}\right\}_{i=1}^{N_{r}}$ by the solutions at $N_{r}$ different time instants of (7), or use the stationary solutions from $E(\mathbf{y}, U)=0$ for different control values. Given the reduced basis space $X_{r}$, we define the reduced-order model by projecting (7): For $\mathbf{y}^{r}=\sum_{i=1}^{N_{r}} \alpha_{i}(t) \boldsymbol{\Phi}_{i}$,

$$
\left(\frac{d \mathbf{y}^{r}}{d t}+E\left(\mathbf{y}^{r}, U\right), \boldsymbol{\Phi}_{i}\right)=0, \quad \mathrm{i}=1, \ldots \ldots, N_{r} .
$$


The dimension of the reduced basis space is very much problem-dependent. The reduced basis elements constructed in the above manner can be nearly linearly dependent. We therefore monitor the condition number of the mass matrix, whose entries are given by

$$
M_{i, j}=\left(\boldsymbol{\Phi}_{i}, \boldsymbol{\Phi}_{j}\right),
$$

and stop adding basis vectors when the condition number exceeds a prescribed value. Our computational experiments and the computations reported for structural problems in the references mentioned earlier seem to indicate that an accurate approximation can be obtained for large range of parameter values using 5 to 10 basis elements. Therefore, the resulting reduced order model is dense but it is small compared to the sparse but large system that result from the standard basis functions.

In our previous calculations, see [12], we only employed Lagrange and Hermite basis due to the following reasons. The system (3) can be ill-posed and one cannot continue to use the same basis elements generated at fixed parameter $\mu^{*}$ to compute solutions when the parameter of interest is significantly away from it. In such cases reduced basis elements have to be updated and the solution is sought in the new reduced basis space. Moreover, generating the right hand side of (3) could be quite complicated in certain problems.

According to our comparison study carried out in [12] for driven cavity flow, the performance of Hermite approach is better than that of Lagrange. The basis elements for the Lagrange approach were selected at Reynolds numbers 100, 300, 500 and 700, and that for the Hermite was selected at 300 and 700 . The comparison was carried out by computing the driven cavity flow at Reynolds number 1200 . The $L_{2}$-norm difference between the full mixed-finite element solution $u_{f}$ and the reduced basis solution using these two approaches are as follows: $\left\|\mathbf{u}_{l}-\mathbf{u}_{f}\right\|_{0}=0.0889$ and $\left\|\mathbf{u}_{h}-\mathbf{u}_{f}\right\|_{0}=0.0766$, where $\mathbf{u}_{l}$ is the solution obtained using Lagrange approach and $\mathbf{u}_{h}$ is that obtained using Hermite approach.

\section{Reduced Order Model for Viscous Flows}

In this section we will develop a reduced order model for the unsteady viscous flows. We consider two dimensional viscous incompressible fluid flow which are governed by the Navier-Stokes equations,

$$
\begin{gathered}
\mathbf{u}_{t}-\frac{1}{R e} \Delta \mathbf{u}+\mathbf{u} \cdot \nabla \mathbf{u}+\nabla p=0, \\
\nabla \cdot \mathbf{u}=0, \quad \mathbf{u}(\mathbf{x}, 0)=\mathbf{u}_{0}(\mathbf{x})
\end{gathered}
$$

in the domain $\Omega \times[0, T]$. Here the velocity $\mathbf{u}$, the pressure $\mathrm{p}$, the time $\mathrm{t}$ and the spatial variable $\mathbf{x}$ are in non-dimensional form. The Reynolds' number Re is defined as $R e=\rho U_{0} L / \mu$, where $\rho$ is the density, $U_{0}$ is the non-dimensional velocity.

We divide the boundary of the domain $\Gamma$ into three parts $\Gamma_{1}, \Gamma_{c}$ and $\Gamma_{\text {out }}$, and impose the following boundary conditions:

$$
\begin{aligned}
\mathbf{u} & =c(t) \boldsymbol{\tau} & & \text { on } & \Gamma_{\mathrm{C}} \times[0, T] \\
p \mathbf{n}-\frac{1}{R e} \frac{\partial \mathbf{u}}{\partial n} & =0 & & \text { on } & \Gamma_{\text {out }} \times[0, T] \\
\mathbf{u} & =\mathbf{u}_{b} & & \text { on } & \Gamma_{1} \times[0, T] .
\end{aligned}
$$

For the controlled problems presented in the sequel the control action is through wall movement on part of the boundary $\Gamma_{c}$. The function $c(t)$ represents velocity on $\Gamma_{c}$ and $\boldsymbol{\tau}$ is the unit tangential vector. Moreover, $\mathbf{u}_{b}$ is a prescribed boundary velocity. 
The boundary condition on $\Gamma_{\text {out }}$ is not "physical" but used to represent the flow in an unbounded region; see [21].

\section{A. Weak Formulation}

For the finite dimensional approximation and for the subsequent reduced order approximation, we need a weak form of the state equations (9). A weak form of the equations (9) s obtained by multiplying both sides of the first and second equations by $\mathbf{v}$ and $q$, respectively, and applying Greens formula; see [24] for similar problems,

$$
\begin{aligned}
\left(\mathbf{u}_{t}+\mathbf{u} \cdot \nabla \mathbf{u}, \mathbf{v}\right)+\frac{1}{R e}(\nabla \mathbf{u}, \nabla \mathbf{v})-(p, \nabla \cdot \mathbf{v}) & =0 \\
(\nabla \cdot \mathbf{u}, q) & =0
\end{aligned}
$$

for all test functions $(\mathbf{v}, q) \in \mathbf{V} \times L^{2}(\Omega)$, where

$$
\mathbf{V}=\left\{\mathbf{v} \in \mathbf{H}^{1}(\Omega):\left.\quad \mathbf{v}\right|_{\Gamma \backslash \Gamma_{\text {out }}}=0\right\} .
$$

The state variables $(\mathbf{u}, p)$ for the problem are taken to be

$$
\begin{gathered}
\mathbf{u} \in L^{2}\left(0, T ; \mathbf{H}^{1}(\Omega)\right),\left.\quad \mathbf{u}\right|_{\Gamma_{C}}=c(t) \boldsymbol{\tau} \text { and }\left.\mathbf{u}\right|_{\Gamma_{1}}=\mathbf{u}_{b}, \\
p \in \mathrm{L}^{2}(\Omega) .
\end{gathered}
$$

When $\int_{\Gamma_{\text {out }}}=0$, for example in the cavity flow presented in a later section, it is usual to replace the pressure space $\mathrm{L}^{2}(\Omega)$ in the weak form by

$$
\mathrm{L}_{0}^{2}(\Omega)=\left\{q \in \mathrm{L}^{2}(\Omega) \mid \int_{\Omega} q d \Omega=0\right\}
$$

to compute pressure uniquely.

\section{B. Finite Element Approximations}

To approximate the solutions of (10), we will use standard mixed finite element method. For this, we write $\mathbf{u}$ and $p$ as linear combination of finite number of basis functions:

$$
\mathbf{u}^{h}=\mathbf{u}_{0}^{h}+\sum_{i=1}^{N_{u}} \mathbf{u}_{i}(t) \Phi_{i}(\mathbf{x}) \quad \text { and } \quad p^{h}=\sum_{i=1}^{N_{p}} p_{i}(t) \Psi(\mathbf{x})
$$

with $\mathbf{u}_{0}^{h}$ be the finite element interpolant of non-zero boundary conditions imposed on u. Then

$$
\begin{aligned}
& \mathbf{V}^{N_{u}}=\operatorname{span}\left\{\Phi_{1}, \Phi_{2}, \ldots \ldots, \Phi_{N_{u}}\right\}, \\
& Q^{N_{p}}=\operatorname{span}\left\{\Psi_{1}, \Psi_{2}, \ldots \ldots, \Psi_{N_{p}}\right\}
\end{aligned}
$$

and $\mathbf{V}^{N_{u}} \times Q^{N_{p}} \subset \mathbf{V} \times L^{2}$. The approximate system is determined by restricting the weak form (10) to $\mathbf{V}^{N_{u}} \times Q^{N_{p}}$ with basis functions $\Phi_{i}$ substituted for the test 
functions $\mathbf{v}$ and the basis functions $\Psi_{i}$ substituted for the test functions $q$. Then the following finite dimensional system results:

$$
\begin{aligned}
& \mathbf{M} \dot{\mathbf{u}}+\mathbf{S u}+\mathbf{N}(\mathbf{u}) \mathbf{u}+\mathbf{L}^{T} \mathbf{p}=\mathbf{F}, \\
& \mathbf{L u}=0
\end{aligned}
$$

where $\mathbf{S}$ is the diffusion or stiffness matrix, $\mathbf{N}$ the convection matrix, $\mathbf{L}$ the continuity matrix, $\mathbf{M}$ the mass matrix and $\dot{\mathbf{u}}=\frac{d \mathbf{u}}{d t}$. Moreover $\mathbf{u}$ and $\mathbf{p}$ are the finite dimensional velocity and pressure, respectively. We call the approximations using standard finite element basis functions such as quadratic or linear piecewise polynomials by "full order methods/discretization" and those using RBM by "reduced order methods". For the full discretization we use continuous piecewise quadratics for the velocity $\mathbf{u}$ and continuous piecewise linear functions for the pressure $p$; the same triangular grid is used for both finite element spaces; This choice of spaces complies with the div-stability condition which is required for stable computation of pressure; see [6].

The nonlinear differential algebraic equations (DAE) (11) is discretized using backward Euler in time with the time step $\Delta t=0.01$ and the resulting nonlinear algebraic system is solved using Newtons method along with a banded Gaussian elimination.

In order to have a test bed to compare the RBM simulations with the 'full order method" simulations, we consider flow through backward-facing step channel. A schematic of the geometry is given in Figure 2. In Figure 2, the height of the inflow boundary is 0.5 and that of the outflow boundary is 1 . The length of the narrower section of the channel is 0.5 and the total length of the channel is 12 .

The following boundary conditions are imposed:

$$
\begin{aligned}
\mathbf{u} & =(8(y-1 / 2)(1-y), 0)=\mathbf{u}_{\text {in }} & & \text { on } & & \Gamma_{\text {in }} \times[0, T] \\
p \mathbf{n}-\frac{1}{R e} \frac{\partial \mathbf{u}}{\partial n} & =(0,0) & & \text { on } & & \Gamma_{\text {out }} \times[0, T] \\
\mathbf{u} & =(0,0) & & \text { on } & & \Gamma_{t} \cup \Gamma_{b} \cup \Gamma_{s} \cup \Gamma_{c} \times[0, T]
\end{aligned}
$$

The computational domain was divided into 682 triangles with finer mesh around the recirculation region. This resulted in a system of 3,032 ordinary differential equations that has to be solved for the unknown coefficients.

It is well known that beyond certain Reynolds' number the flow separates and a recirculation forms near the corner region. We carried out simulations at a Reynolds' number of 1000 and the long term flow simulation is given in Figure 3. It clearly predicts the re-circulations first near the corner of the step and the second one near the wall opposite to the step.

\section{ROM in Flow Simulation}

We will demonstrate the performance of the ROM for the unsteady viscous flows on the backward-facing step channel flow problem. We follow the Lagrange approach for ROM simulations. The basis elements in the Lagrange subspace are snapshots of the problem obtained by solving the system (9) using a full order method; see Figure 1. Supposing $\left\{\Psi_{i}\right\}_{i=1}^{N_{r}}$ denote the snapshots, the reduced order subspace is defined as $V^{R B M}=\operatorname{span}\left\{\Psi_{i}\right\}_{i=1}^{N_{r}}$. Once we have a reduced order subspace $V^{R B M}$, the system (9) is projected onto $V^{R B M}$ to obtain a reduced order model.

The construction of ROM involves the following steps: 
(I) Solve the state equation (11) at $\mathrm{N}$ different time steps and obtain "snapshots" $\mathcal{S}$; see Figure 1.

(II) $\operatorname{Set} \mathbf{V}^{R B M}=\operatorname{span}\left\{\Psi_{1}, \Psi_{2}, \ldots \ldots \ldots, \Psi_{N_{r}}\right\}$. And set $\mathbf{u}^{r}=\Psi_{0}+\sum_{i=1}^{N_{r}} \alpha_{i}(t) \Psi_{i}$, where $\Psi_{0}$ account for the nonzero boundary values.

(III) Restrict the weak form (10) to $\mathbf{V}^{R B M}$ and solve for $\alpha_{i}, i=1,2, \ldots ., N_{r}$.

(IV) Set $\mathbf{u}^{r}=\Psi_{0}(\mathbf{x})+\sum_{i=1}^{N_{r}} \alpha_{i}(t) \Psi_{i}$.

In order to derive the reduced order model, let us choose $N_{r}$ and expand the solution as

$$
\mathbf{u}^{r}=\boldsymbol{\Phi}_{0}(\mathbf{x})+\sum_{i=1}^{N_{r}} \alpha_{i}(t) \Phi_{i}(\mathbf{x}) .
$$

Then the Galerkin approximation of the weak form (10) is as follows

$$
\left(\mathbf{u}_{t}^{r}+\mathbf{u}^{r} \cdot \nabla \mathbf{u}^{r}, \boldsymbol{\Phi}_{i}\right)-\left(p^{r}, \nabla \cdot \boldsymbol{\Phi}_{i}\right)+\frac{1}{R e}\left(\nabla \mathbf{u}^{r}, \nabla \boldsymbol{\Phi}_{i}\right)+\left(p^{r} \mathbf{n}-\frac{1}{R e} \frac{\partial \mathbf{u}^{r}}{\partial \mathbf{n}}, \boldsymbol{\Phi}_{i}\right)_{\Gamma_{\text {out }}}=0,
$$

for all $\Phi_{i} \in \mathbf{V}^{R B M}$. At this point it is important to note that the basis functions $\left\{\boldsymbol{\Phi}_{i}\right\}_{i=1}^{N_{r}}$ are divergence free as flow is incompressible and satisfy zero boundary conditions on $\Gamma \backslash \Gamma_{\text {out }}$. Using these properties of $\left\{\boldsymbol{\Phi}_{i}\right\}_{i=1}^{N_{r}}$ and the boundary condition on $\Gamma_{\text {out }}$, we see that the pressure term and the boundary term vanishes. Then (13) reduces to

$$
\left(\mathbf{u}_{t}^{r}+\mathbf{u}^{r} \cdot \nabla \mathbf{u}^{r}, \Phi_{i}\right)+\frac{1}{R e}\left(\nabla \mathbf{u}^{r}, \nabla \boldsymbol{\Phi}_{i}\right)=0,
$$

for all $\Phi_{i} \in \mathbf{V}^{R B M}$. On substitution of (12) into (14) we obtain the following nonlinear evolution equation for the coefficients $\alpha_{i}(t)$ :

$$
\begin{aligned}
& M \dot{\boldsymbol{\alpha}}+\mathcal{A} \widehat{\boldsymbol{\alpha}}+\widehat{\boldsymbol{\alpha}}^{T} \mathcal{N} \widehat{\boldsymbol{\alpha}}=0, \\
& M \boldsymbol{\alpha}(0)=\boldsymbol{\alpha}_{0},
\end{aligned}
$$

where $\boldsymbol{\alpha}=\left(\alpha_{1}, \alpha_{2}, \ldots, \alpha_{N_{r}}\right)^{T}, \widehat{\boldsymbol{\alpha}}=(1, \alpha)^{T}$ and we define the mass matrix M, stiffness matrix $\mathrm{A}$ and the initial condition $\boldsymbol{\alpha}_{0}$ as follows:

$$
\begin{gathered}
M_{i j}=\left(\boldsymbol{\Phi}_{i}, \boldsymbol{\Phi}_{j}\right), \quad i, j=1, \ldots, N_{r}, \\
\mathcal{A}_{i j}=\frac{1}{R e}\left(\nabla \boldsymbol{\Phi}_{i}, \nabla \boldsymbol{\Phi}_{j}\right), \quad i=1, \ldots ., N_{r} \quad j=0, \ldots, N_{r} \\
\alpha_{0 i}=\left(\mathbf{u}_{0}, \Phi_{i}\right), \quad i=1, \ldots, N_{r},
\end{gathered}
$$

where $\mathbf{u}_{0}$ is the initial condition. Moreover, $\left(\widehat{\boldsymbol{\alpha}}^{T} \mathcal{N} \widehat{\boldsymbol{\alpha}}\right)_{i}=\widehat{\boldsymbol{\alpha}}^{T} \mathcal{P}_{i} \widehat{\boldsymbol{\alpha}}, \quad i=1, \ldots, N_{r}$, and

$$
\left(\mathcal{P}_{i}\right)_{k l}=\left(\boldsymbol{\Phi}_{k} \cdot \nabla \boldsymbol{\Phi}_{l}, \boldsymbol{\Phi}_{i}\right), \quad i=1, \ldots, N_{r} \quad k, l=0, \ldots, N_{r} .
$$


The solution to the above initial value problem (15) was obtained using an implicit Euler method for the coefficients of the RBM approximation.

\section{Numerical Results}

We selected $\left\{t_{i}\right\}_{i=1}^{N_{r}}$ at eleven regular time instances in the time interval $[0,10]$ and solved the system (9) to obtain the snapshots $\left\{\Psi_{i}\right\}_{i=1}^{N_{r}+1}$, we define the basis elements as $\left\{\Phi_{i}\right\}_{i=1}^{N_{r}}=\left\{\Psi_{i+1}-\Psi_{i}\right\}_{i=1}^{N_{r}}$ and the reduced order solution $\mathbf{u}^{r}$ is defined by $\mathbf{u}^{r}=\Psi_{N_{r}+1}+\sum_{i=1}^{N_{r}} \alpha_{i} \Phi_{i}$.

The initial value problem (15) for the nonlinear ODE was solved using backward Euler method with the time step $\Delta t=10^{-3}$ and the resulting nonlinear algebraic system was solved using Newton iterative method. As each Newton step involves small linear system, it was solved using Gaussian elimination. The Figures $3-4$ are the channel flow computations with "full order solution" and reduced order solution at time $\mathrm{t}=10$ which shows excellent qualitative agreement.

\section{ROM for Optimal Control of Viscous Flows}

In this section we turn to the application of the reduced basis method for the derivation of reduced order control problems. We first present a general framework for the optimal control problems to be followed. Then we will present reduced order control problems and outline a method based on the necessary conditions of optimality to solve such problems.

Let us first introduce the control problem for the unsteady viscous flows in the following abstract form:

(P) Minimize $\mathcal{J}(\mathbf{u}, U)=\int_{0}^{T} \frac{1}{2}\left[\mathcal{F}(\mathbf{u})+\beta|U|^{2}\right] d t \quad$ subject to $(9 a)-(9 b)$,

where $c(t) \boldsymbol{\tau}, c:[0, T] \rightarrow \mathbb{R}$ is the control input and influences the state only through part of the boundary $\Gamma_{c}$. The specific form of $\boldsymbol{\tau}$ for the cavity and channel problems will become clear later. Moreover, the positive parameter $\beta$ in the cost functional $\mathcal{J}$ is the penalty term and the inclusion of $U=\dot{c}$ in it makes sure the rate of change of control velocity is minimized.

We will study two control problems that fall into the framework of $(\mathbf{P})$ :

(P1) The velocity tracking problem in cavity flow with the cost function $J$ defined with

$$
\mathcal{F}(\mathbf{u})=\int_{\Omega}\left|\mathbf{u}-\mathbf{u}_{d}\right|^{2} d \Omega
$$

(P2) The vorticity reduction problem in channel flow with the cost function $J$ defined with

$$
\mathcal{F}(\mathbf{u})=\int_{\Omega}|\nabla \times \mathbf{u}|^{2} d \Omega
$$

\section{A. Reduced Order Control Problem}

For the boundary control problem described above the reduced order solution $\mathbf{u}^{r}$ is given by

$$
\mathbf{u}^{r}=\boldsymbol{\Phi}_{0}(\mathbf{x})+c(t) \boldsymbol{\Phi}_{N_{r+1}}(\mathbf{x})+\sum_{i=1}^{N_{r}} \boldsymbol{\alpha}_{i}(t) \boldsymbol{\Phi}_{i}(\mathbf{x})
$$


where $\boldsymbol{\Phi}_{0}$ corresponds to the fixed boundary conditions, for example inflow conditions. Moreover, $\{\boldsymbol{\Phi}\}_{i=1}^{N_{r}}$ are the test functions satisfying the homogeneous boundary conditions and $\boldsymbol{\Phi}_{i_{N_{r+1}}}$ is the trial function corresponding to the control force. The number of test functions $N_{r}$ should be chosen large enough to accurately capture the dynamics of the system and small enough not to make the system ill-conditioned.

Inserting the expansion (16) into the Galerkin projection (14) of the Navier-Stokes equations, we obtain

$$
\begin{aligned}
& M \dot{\boldsymbol{\alpha}}+A \mathbf{X}+\mathbf{X}^{T} \mathcal{N} \mathbf{X}+\mathbf{b} U=0, \\
& \dot{c}=U, \\
& M \boldsymbol{\alpha}(0)=\boldsymbol{\alpha}_{0},
\end{aligned}
$$

where $\mathbf{X}=(1, \boldsymbol{\alpha}, c)^{T}, U$ is the control, and we define the mass matrix $\mathrm{M}$, stiffness matrix $\mathrm{A}$ and the initial condition $\boldsymbol{\alpha}_{0}$ as follows:

$$
\begin{aligned}
& M_{i j}=\left(\boldsymbol{\Phi}_{i}, \boldsymbol{\Phi}_{j}\right), \quad i, j=1, \ldots, N_{r}, \\
& \mathcal{A}_{i j}=\frac{1}{\operatorname{Re}}\left(\nabla \boldsymbol{\Phi}_{i}, \nabla \boldsymbol{\Phi}_{j}\right), \quad i=1, \ldots, N_{r} \quad j=0, \ldots, N_{r+1} \\
& \alpha_{0 i}=\left(\mathbf{u}_{0}, \Phi_{i}\right), \quad i=1, \ldots, N_{r},
\end{aligned}
$$

where $\mathbf{u}_{0}$ is the initial condition. Moreover, $\left(\mathbf{X}^{T} \mathcal{N} \mathbf{X}\right)_{i}=\mathbf{X}^{T} \mathcal{P}_{i} \mathbf{X}, \quad i=1, \ldots, N_{r}$, and

$$
\left(\mathcal{P}_{i}\right)_{k l}=\left(\boldsymbol{\Phi}_{k} \cdot \nabla \boldsymbol{\Phi}_{l}, \boldsymbol{\Phi}_{i}\right), \quad i=1, \ldots, N_{r} \quad k, l=0, \ldots, N_{r+1} .
$$

We define the reduced order control problem as follows:

$$
\text { Minimize } \mathcal{J}(\mathbf{X}, U)=\int_{0}^{T}\left[\frac{1}{2} \mathbf{X}^{T} \mathcal{Q} \mathbf{X}-\mathbf{X} \cdot \mathbf{d}+\epsilon+\frac{\beta}{2} U^{2}\right] d t
$$

subject to

$\left(\mathbf{P}^{\mathbf{r}}\right)$

$$
\left\{\begin{array}{l}
M \dot{\boldsymbol{\alpha}}+A \mathbf{X}+\mathbf{X}^{T} \mathcal{N} \mathbf{X}+\mathbf{b} U=0 \\
\dot{c}=U \\
M \boldsymbol{\alpha}(0)=\boldsymbol{\alpha}_{0}
\end{array}\right.
$$

where $\mathbf{b}_{i}=\left(\mathbf{u}_{c}, \boldsymbol{\Phi}_{i}\right), i=1, \ldots, N_{r}$. Moreover, when $\mathcal{F}(\mathbf{u})=\mathcal{F}_{\text {tracking: }}$ :

$$
\mathcal{Q}_{i, j}=\left(\boldsymbol{\Phi}_{i}, \boldsymbol{\Phi}_{j}\right), \quad i, j=0, \ldots, N_{r+1}, \quad \mathbf{d}_{i}=\left(\mathbf{u}_{d}, \boldsymbol{\Phi}_{i}\right), \quad i=1, \ldots, N_{r} \text { and } e=\frac{1}{2}\left|\mathbf{u}_{d}\right|^{2},
$$

and when $\mathcal{F}(\mathbf{u})=\mathcal{F}_{\text {vorticity }}$

$$
\mathcal{Q}_{i, j}=\left(\nabla \times \boldsymbol{\Phi}_{i}, \nabla \times \boldsymbol{\Phi}_{j}\right), \quad \mathbf{d}_{i}=\epsilon=0, \quad i, j=0, \ldots \ldots, N_{r+1} .
$$

We note here that we can cast this optimal control problem in a generic form as

$$
\text { Minimize } \mathcal{J}(\mathbf{X}, U)=\int_{0}^{T}[\ell(\mathbf{X})+h(U)] d t
$$


subject to

$$
\begin{aligned}
& \dot{\mathbf{X}}=f(\mathbf{X})+B U, \\
& \mathbf{X}(0)=\mathbf{X}_{0} .
\end{aligned}
$$

Letting $h(U)=\frac{\beta}{2} U^{2}$, we can write down the necessary conditions of optimality as

$$
\left.\begin{array}{l}
\dot{\mathbf{X}}=f(\mathbf{X})+B U, \quad \mathbf{X}(0)=\mathbf{X}_{0} \\
-\dot{\boldsymbol{\zeta}}=f_{\mathbf{X}}(\mathbf{X})^{T} \boldsymbol{\zeta}(t)+\ell_{\mathbf{X}}(\mathbf{X}), \quad \boldsymbol{\zeta}(T)=0 \\
U=-\frac{1}{\beta} B^{T} \boldsymbol{\zeta}
\end{array}\right\}
$$

which constitutes the well-known pontryagin maximum principle and $\boldsymbol{\zeta}$ is the Lagrange multiplier or the adjoint variable. Thus the optimal control $U$ for $(17)-(18)$ is given by $U=-\frac{1}{\beta} B^{T} \boldsymbol{\zeta}$.

\section{B. Solution Procedure for Reduced Order Control Problem}

In what follows, we describe a general procedure for solving the two point boundary value problem (TPBV) (19). Instead of the direct approximation of the TPBV, we will approximate (17)-(18) using the Crank-Nicholson method for the constraint and the trapezoidal rule for the cost functional. We obtain

$$
\text { Minimize } \quad \mathcal{J}^{N}=\sum_{k=1}^{N}\left[\frac{1}{2}\left(\ell\left(\mathbf{X}^{k-1}\right)+\ell\left(\mathbf{X}^{k}\right)\right)+h\left(U^{k}\right)\right] \Delta t
$$

subject to

$$
\frac{\mathbf{X}^{k}-\mathbf{X}^{k-1}}{\Delta t}=\frac{1}{2}\left(f\left(\mathbf{X}^{k}\right)+f\left(\mathbf{X}^{k-1}\right)\right)+B U^{k}, \quad k=1, \ldots \ldots, N
$$

where $N \Delta t=T$ and $\mathbf{X}^{0}=\mathbf{X}_{0}$. The necessary optimality condition for (20)-(21) is given by

$$
\left.\begin{array}{l}
\frac{\mathbf{X}^{k}-\mathbf{X}^{k-1}}{\Delta t}=\frac{1}{2}\left(f\left(\mathbf{X}^{k}\right)+f\left(\mathbf{X}^{k-1}\right)\right)-\frac{1}{\beta} B B^{T} \boldsymbol{\zeta}^{k} \\
-\frac{\boldsymbol{\zeta}^{k+1}-\boldsymbol{\zeta}^{k}}{\Delta t}=\frac{1}{2} f_{\mathbf{X}}\left(\mathbf{X}^{k}\right)^{T}\left(\boldsymbol{\zeta}^{k}+\boldsymbol{\zeta}^{k+1}\right)+\ell_{\mathbf{X}}\left(\mathbf{X}^{k}\right)
\end{array}\right\}
$$

for $k=1, \ldots ., N$ and $\mathbf{X}^{0}=\mathbf{X}_{0}$ and $\boldsymbol{\zeta}^{N+1}=0$, and the optimal control to (20)-(21) is given by

$$
U^{k}=-\frac{1}{\beta} B^{T} \boldsymbol{\zeta}^{k}
$$

The system (22)-(23), which is an approximation to the TPBV (19), is a sparse system of nonlinear equations in $\mathbf{X}^{k}, \boldsymbol{\zeta}^{k}$ and can be solved using the Newtons method:

$$
\mathcal{F}\left(\mathbf{Y}^{-}\right)\left(\mathbf{Y}^{+}-\mathbf{Y}^{-}\right)=\mathcal{F}\left(\mathbf{Y}^{-}\right)
$$


where $\mathbf{Y}=\left(\mathbf{X}^{1}, \mathbf{X}^{2}, \ldots, \mathbf{X}^{N}, \boldsymbol{\zeta}^{1}, \boldsymbol{\zeta}^{2}, \ldots \ldots, \boldsymbol{\zeta}^{N}\right)$,

$$
\mathcal{F}(\mathbf{Y})=\left[\begin{array}{l}
\frac{\mathbf{X}^{k}-\mathbf{X}^{k-1}}{\Delta t}-\frac{1}{2}\left(f\left(\mathbf{X}^{k}\right)+f\left(\mathbf{X}^{k-1}\right)\right)+\frac{1}{\beta} B B^{T} \boldsymbol{\zeta}^{k} \\
\frac{\boldsymbol{\zeta}^{k+1}-\boldsymbol{\zeta}^{k}}{\Delta t}+\frac{1}{2} f_{\mathbf{X}}\left(\mathbf{X}^{k}\right)^{T}\left(\boldsymbol{\zeta}^{k}+\boldsymbol{\zeta}^{k+1}\right)+\ell_{\mathbf{X}}\left(\mathbf{X}^{k}\right)
\end{array}\right]
$$

and the Jacobian has the following form

$$
\mathcal{F}^{\prime}=\left[\begin{array}{cc}
A & S \\
Q & -A^{T}
\end{array}\right]
$$

where $\mathrm{A}$ is block lower bi-diagonal, and $\mathrm{S}$ and $\mathrm{Q}$ are block diagonal with block size $\mathrm{M}$. The diagonal and sub-diagonal blocks of $\mathrm{A}$ are given by

$$
A_{k, k}=\frac{I}{\Delta t}+\frac{1}{2} f_{\mathbf{X}}\left(\mathbf{X}^{k}\right) \quad \text { and } \quad A_{k+1, k}=-\frac{I}{\Delta t}+\frac{1}{2} f_{\mathbf{X}}\left(\mathbf{X}^{k}\right) .
$$

The block $S$ is the constant

$$
S_{k, k}=\frac{1}{\beta} B^{T} B,
$$

and the diagonal block $Q$ is given by

$$
Q_{k, k}=\ell_{\mathbf{X X X}}\left(\mathbf{X}^{k}\right)+\frac{1}{2} f_{\mathbf{X}, \mathbf{X}}\left(\mathbf{X}^{k}\right)^{T}\left(\boldsymbol{\zeta}^{k}+\boldsymbol{\zeta}^{k+1}\right) .
$$

We note here that the vector $\mathbf{Y}=\left(\mathbf{X}^{1}, \mathbf{X}^{2}, \ldots \ldots, \mathbf{X}^{N}, \boldsymbol{\zeta}^{1}, \boldsymbol{\zeta}^{2}, \ldots \ldots, \boldsymbol{\zeta}^{N}\right)^{T}$ is rearranged to $\mathbf{Y}=\left(\mathbf{X}^{1}, \boldsymbol{\zeta}^{1}, \mathbf{X}^{2}, \boldsymbol{\zeta}^{2}, \ldots \ldots, \mathbf{X}^{N}, \boldsymbol{\zeta}^{N}\right)^{T}$ then each Newton step can be solved using a block tridiagonal algorithm.

\section{Computational Results for Control Problems}

In this section we will show the viability of the reduced order model for control problems by implementing it computationally on cavity and channel flows.

\section{A. Example I: Driven Cavity Flow}

The first situation we consider is that of driven cavity as depicted in Figure 5. Traditionally, the problem is to find the two dimensional motion of a fluid confined in the cavity when only top surface moves with a velocity.

The problem we are interested in is: Given velocity profile (desired) $\mathbf{u}_{d}$ inside the cavity, can we find the bottom velocity $c(t)$ such that the fluid velocity $\mathbf{u}$ is driven to the desired one?

This is a control problem that can be posed as a minimization problem with the cost function

$$
\mathcal{J}(\mathbf{u})=\int_{0}^{T} \int_{\Omega}\left|\mathbf{u}-\mathbf{u}_{d}\right|^{2} d \Omega d t
$$

subject to the constraint that the fluid obeys the equation of motion $(9 a)$ and boundary conditions:

$$
\left.\mathbf{u}\right|_{\Gamma_{\boldsymbol{t}}}=(1,0),\left.\quad \mathbf{u}\right|_{\Gamma_{\boldsymbol{b}}}=(c(t), 0), \quad \text { and }\left.\quad \mathbf{u}\right|_{\Gamma_{s}}=(0,0) .
$$


We used the reduced basis method introduced in the previous section to approximate this control problem. The basis functions $\left\{\mathbf{u}_{i}: \quad i=1, \ldots \ldots, N_{r+2}=11\right\}$ were chosen as solutions to the stationary Navier-Stokes equations with the boundary conditions as shown in Table 1 for the driven cavity. We used a $29 \times 29$ nonuniform mesh with corner refinement and the Reynolds number of $R e=500$. The uncontrolled flow is shown in Figure 6 where the top velocity $u=1$ and the bottom velocity $u=0$.

We define the reduced order solution as

$$
\mathbf{u}^{r}=\boldsymbol{\Phi}_{0}+c(t) \boldsymbol{\Phi}_{N_{r}+1}+\sum_{i=1}^{N_{r}} \boldsymbol{\alpha}_{i}(t) \boldsymbol{\Phi}_{i},
$$

where the test functions $\boldsymbol{\Phi}_{i}, \quad i=1, \ldots, N_{r}$ are defined such that they satisfy all the Dirichlet boundary conditions and $\boldsymbol{\Phi}_{0}=\mathbf{u}_{1}$ and $\boldsymbol{\Phi}_{N_{r}+1}=\mathbf{u}_{11}$. In order to have convergence, the number of trial functions should be chosen large enough and small enough that it does not make the system ill-posed.

We carried out computations with $N_{r}=4,6$ and 9. The test functions $\boldsymbol{\Phi}_{i}, \quad i=$ $1, \ldots ., N_{r}$, are defined as usual. For example in the case of $N_{r}=4$, we defined them as:

$$
\begin{aligned}
& \boldsymbol{\Phi}_{0}=\mathbf{u}_{1} \\
& \boldsymbol{\Phi}_{1}=\mathbf{u}_{3}-\mathbf{u}_{1}-\mathbf{u}_{11} \\
& \boldsymbol{\Phi}_{2}=\mathbf{u}_{8}-\mathbf{u}_{1}+\mathbf{u}_{11} \\
& \boldsymbol{\Phi}_{3}=\mathbf{u}_{6}-\mathbf{u}_{1}-0.5 \mathbf{u}_{11} \\
& \boldsymbol{\Phi}_{4}=\mathbf{u}_{2}-\mathbf{u}_{1}+0.5 \mathbf{u}_{11} \\
& \boldsymbol{\Phi}_{5}=\mathbf{u}_{11} .
\end{aligned}
$$

The desired flow field $\mathbf{u}_{d}$ was taken to be the one shown in Figure 7 which was obtained by computing the steady driven cavity flow with constant bottom velocity $u=1.5$.

The control problem is to find the time evolution of the control input $c(t)$ (bottom wall velocity) such that the flow field matches as close as possible the desired velocity $\mathbf{u}_{d}$. We solved the control problem using the methods described in the previous sections in the time interval $[0,10]$. Using the parameters $\Delta t=0.01, \beta=\frac{1}{50}$ and an initial condition $\mathbf{X}_{0}=0$, we obtained convergence in 7 iterations. The resulting optimal control input $c(t)$ with three different number of basis elements are shown in Figure 8 which shows convergence when $N_{r}=9$. We also computed the flow field with the control input for the driven cavity and the resulting flow agrees well with the desired flow.

\section{B. Example II: Channel Flow}

The second situation is that of channel flows depicted in Figures 2 and 12 . In the first configuration we consider is a backward-facing step and assume the flow is parabolic (Poiseuille flow) with $\mathbf{u}_{\text {in }}=8(y-1 / 2)(1-y)$. There is a recirculation region in the corner whose size increases with Reynolds number. Figure 9 qualitatively shows it for large Reynolds number. In the second configuration we consider a forward-facing step and assume that the inflow parabolic with $\mathbf{u}_{\text {in }}=y(1-y / 3) / 3$. In this case, there is a recirculation on the top of the step. Figure 13 qualitatively demonstrates the situation for high Reynolds numbers.

The problem we are interested in both configurations is: Can we shape the flow to obtain a flow which is free of recirculation by means of controlled movement of wall along some part of the boundary? 
This is a control problem that can be cast a minimization problem with the cost function

$$
\mathcal{J}(\mathbf{u})=\int_{0}^{T} \int_{\Omega}|\nabla \times \mathbf{u}|^{2} d \Omega
$$

subject to the constraint that the fluid obeys the equation of motion $(9 a)$ and boundary conditions:

$$
\begin{aligned}
\mathbf{u} & =\mathbf{u}_{\text {in }} & & \text { on } & & \Gamma_{\text {in }} \times[0, T] \\
p \mathbf{n}-\frac{1}{R e} \frac{\partial \mathbf{u}}{\partial n} & =(0,0) & & \text { on } & & \Gamma_{\text {out }} \times[0, T] \\
\mathbf{u} & =c(t) \boldsymbol{\tau} & & \text { on } & & \Gamma_{c} \times[0, T] \\
\mathbf{u} & =(0,0) & & \text { on } & & \Gamma_{t} \cup \Gamma_{b} \cup \Gamma_{s} \times[0, T] .
\end{aligned}
$$

where $\Gamma_{c}$ is part of the boundary where boundary surface is moving (control input); see Figure 2 and Figure 12. Also, $c(t)$ is the magnitude of the boundary surface velocity. In the two channel geometries considered our choice of control portion $\Gamma_{c}$ is not the only one possible. But it is motivated by the fact that if one wants maximum influence in the flow, then the control has to be applied in that vicinity.

\section{Test I: Backward-Facing-Step Channel Flow}

The geometry of the problem along with the boundary conditions is discussed in $\S I I I$ B. The control portion $\Gamma_{c}$ is taken to be the vertical part of the step, i.e., it is the line segment between $y=0$ and $y=0.5$ at $x=1$. Also, we take $\boldsymbol{\tau}=(1,0)$, that is the movement of the wall is vertical and thus $c(t) \in \mathbb{R}$ completely determines the control input.

The computational domain was divided into 794 triangles with finer mesh around the recirculation region and the Reynolds number was taken to be $R e=200$. The uncontrolled flow is shown in Figure 9. The basis functions $\left\{\mathbf{u}_{i}: i=1, \ldots \ldots, N_{r+2}=11\right\}$ were chosen as solutions to the stationary Navier-Stokes equations with the boundary conditions as shown in Table 2 for the backward-facing step channel.

We define the reduced order solution as

$$
\mathbf{u}^{r}=\boldsymbol{\Phi}_{0}+c(t) \boldsymbol{\Phi}_{N_{r}+1}+\sum_{i=1}^{N_{r}} \boldsymbol{\alpha}_{i}(t) \boldsymbol{\Phi}_{i},
$$

where the test functions $\boldsymbol{\Phi}_{i}, \quad i=1, \ldots, N_{r}$ are defined such that they satisfy all the Dirichlet boundary conditions and $\boldsymbol{\Phi}_{0}=\mathbf{u}_{1}$. In order to verify convergence, we carried out computations with $N_{r}=4,6$ and 9 . The test functions $\boldsymbol{\Phi}_{i}, \quad i=1, \ldots \ldots, N_{r}$, are defined as usual. For example in the case of $N_{r}=4$, we defined them as:

$$
\begin{aligned}
& \boldsymbol{\Phi}_{0}=\mathbf{u}_{1} \\
& \boldsymbol{\Phi}_{1}=\mathbf{u}_{6}-2 \mathbf{u}_{4}+\mathbf{u}_{1} \\
& \boldsymbol{\Phi}_{2}=\mathbf{u}_{7}-3 \mathbf{u}_{4}+2 \mathbf{u}_{1} \\
& \boldsymbol{\Phi}_{3}=\mathbf{u}_{8}-4 \mathbf{u}_{4}+3 \mathbf{u}_{1} \\
& \boldsymbol{\Phi}_{4}=\mathbf{u}_{9}-5 \mathbf{u}_{4}+4 \mathbf{u}_{1} \\
& \boldsymbol{\Phi}_{5}=\mathbf{u}_{4}-\mathbf{u}_{1} .
\end{aligned}
$$

We solved the control problem using the methods described in the previous sections in the time interval $[0,100]$. Using the parameters $\Delta t=0.01, \beta=\frac{1}{50}$ and an initial condition $\mathbf{X}_{0}=0$, we obtained convergence in 7 iterations. The initial and final cost 
functional values are 0.59 and 0.43 , respectively. The resulting optimal control input $c(t)$ for three different number of basis elements are shown in Figure 11 which shows convergence when $N_{r}=9$. We also computed the flow field with the control input for the channel and the resulting flow shown in Figure 10 indicates significant reduction in the corner circulation. We also simulated the flow corresponding to the optimal control computed from the reduced order model and the result is shown in Figure 9. The results show significant reduction in the corner circulation.

\section{Test II: Forward-Facing Step Channel Flow}

A schematic of the forward-facing step channel geometry is shown in Figure 12. There the height of the inflow boundary is 3 and that of the outflow boundary is 2. The length of the narrower section of the channel is 1 and the total length is 12 . The computational domain was divided into 582 triangles with finer mesh around the recirculation region. Figure 13 qualitatively demonstrate the situation for Reynolds number of $\operatorname{Re}=1000$. Our objective in this case is to remove the recirculation that occurs on the top of the step. We take the control region $\Gamma_{c}$ to be the line segment between $x=1$ and $x=5$ at $y=1$ here we note that at $x=1$ is where the channel changes its cross section area. Also, we take $\boldsymbol{\tau}=(1,0)$, that is the movement of the wall is horizontal and like in the previous case $c(t) \in \mathbb{R}$ completely determines the control input. The basis functions $\left\{\mathbf{u}_{i}: \quad i=1, \ldots \ldots, N_{r+2}=11\right\}$ were chosen as solutions to the stationary Navier-Stokes equations with the boundary conditions as shown in Table 3 for the forward-facing step channel.

We define the reduced order solution as

$$
\mathbf{u}^{r}=\boldsymbol{\Phi}_{0}+c(t) \boldsymbol{\Phi}_{N_{r}+1}+\sum_{i=1}^{N_{r}} \boldsymbol{\alpha}_{i}(t) \boldsymbol{\Phi}_{i},
$$

where the test functions $\boldsymbol{\Phi}_{i}, \quad i=1, \ldots, N_{r}$ are defined such that they satisfy all the Dirichlet boundary conditions and $\boldsymbol{\Phi}_{0}=\mathbf{u}_{1}$. In order to verify convergence, we carried out computations with $N_{r}=4,6$ and 9 . The test functions $\boldsymbol{\Phi}_{i}, \quad i=1, \ldots \ldots, N_{r}$, are defined as usual. For example in the case of $N_{r}=4$, we defined them as:

$$
\begin{aligned}
& \boldsymbol{\Phi}_{0}=\mathbf{u}_{1} \\
& \boldsymbol{\Phi}_{1}=\mathbf{u}_{11}-3 \mathbf{u}_{5}+2 \mathbf{u}_{1} \\
& \boldsymbol{\Phi}_{2}=\mathbf{u}_{11}-2 \mathbf{u}_{7}+\mathbf{u}_{1} \\
& \boldsymbol{\Phi}_{3}=\mathbf{u}_{11}-1.5 \mathbf{u}_{9}+0.5 \mathbf{u}_{1} \\
& \boldsymbol{\Phi}_{4}=\mathbf{u}_{11}-1.2 \mathbf{u}_{10}+0.2 \mathbf{u}_{1} \\
& \boldsymbol{\Phi}_{5}=10\left(\mathbf{u}_{11}-\mathbf{u}_{1}\right) / 3 .
\end{aligned}
$$

For the vorticity cost, with the Reynolds number 1000 , we solved the reduced order control problem in the time interval $[0,500]$ with the following parameters: $\mathbf{X}_{0}=0$, $\Delta t=0.01$ and $\beta=1 / 50$. Convergence was obtained in 7 iterations and the optimal control $c(t)$ is shown in Figure 15 for two different number of basis elements and convergence was seen with only six basis elements. The initial and final cost functional values are 0.55 and 0.451 , respectively. We also simulated the flow with the optimal control input and the resulting flow given in Figure 14 shows significant reduction in the recirculation.

\section{Conclusions}

In this article, we have presented the reduced basis method and the reduced order modeling approach for optimal control of unsteady flows. In particular the so-called 
Lagrange subspace was used to define reduced order models as it uses numerical simulation to construct its bases. We have shown two different ways to define this subspace: (i) solutions to the time dependent Navier-Stokes equations at various time instances, (ii) solutions to the stationary Navier-Stokes equations with varying boundary velocity control force. The former is used only for simulation of the uncontrolled system as it may not produce a reduced order model which is representative of the control system. However, the latter approach leads more naturally to the reduced order control problem. The reduced order models developed shows it can capture the essential physics and allows very cheap calculations. However, these are not provided as generic methods rather they must be used with care. Whenever they are effective they can provide significant performance with substantially lower on-line computational resources.

\section{Acknowledgments}

This work was supported in part by the Air Force Office of Scientific Research under grants AFOSR F49620-95-1-0437 and AFOSR F49620-95-1-0447. 


\section{References}

[1] B.O. Almroth, "Automatic choice of global Shape functions in structural analysis", AIAA Journal, Vol. 16, 1978, pp. 525-528.

[2] G. Barwolff, H. Wengle and H. Jeggle, "Direct Numerical Simulation of transitional backwardfacing step flow manipulated by oscillating blowing/suction" Engineering Turbulence Modeling and Experiments, edited by W. Rodi and G. Bergeles, Vol. 3, 1996, pp. 219-228.

[3] F.K. Bhatttacharjee, B. Scheelke and T.R. Troutt, "Modification of vortex interactions in a re-attaching separated flow", AIAA J., Vol. 24, 1986, pp. 623-629.

[4] J. A. Burns and Y.-R. Ou, "Feedback control of the driven cavity problem using LQR Designs", Proc. 33rd IEEE Conference on Decision and Control, Florida, 1994, pp. 289-294.

[5] H. O. Fattorini and S. S. Sritharan, "Existence of optimal controls for viscous flow problems", Proc. Royal Society of London A, Vol. 439, 1992, pp. 81-102.

[6] M. D. Gunzburger, Finite Element Methods for Viscous Incompressible Flows, Academic Press, Inc. London, 1989.

[7] M. D. Gunzburger, L. S. Hou and T. P. Svobodny, "Boundary velocity control of incompressible flow with an application to viscous drag reduction", SIAM J. Control and Optimization, Vol. 30, 1992, pp. 167-181.

[8] M. A. Z. Hasan, "The flow over backward-facing step under controlled perturbation", J. of Fluid Mechanics, Vol. 238, 1992, pp. 73-96.

[9] L. S. Hou and S. S. Ravindran, "A penalized Neumann control approach for solving an optimal Dirichlet control problem for the Navier-Stokes equations", SIAM Journal of Control and Optimization, Vol. 36, No. 5, 1997, pp. 1795-1814.

[10] - "Computations of boundary optimal control problems for an electrically conducting fluid", Journal of Computational Physics, Vol. 128, No.2, 1996, pp. 319-330.

[11] K. Ito and S. S. Ravindran,, "Optimal control of thermally convected fluid flows", SIAM J. Scientific Computing, Vol. 19, No. 6, 1998, pp. 1847-1869.

[12] - "A Reduced Order Method for Simulation and Control of Fluid Flows", Journal of Computational Physics, Vol. 143, 1998, pp. 403-425.

[13] M. Kiya, M. Shimizu and O. Mochizuki, "Sinusoidal forcing of a turbulent separation bubble", J. Fluid Mechanics, Vol. 342, 1997, pp. 119-139.

[14] V. J. Modi, F. Mokhtarian, M. Fernando and T. Yokomizo, "Moving surface boundary-layer control as applied to two-dimensional airfoils", J. Aircraft, Vol. 28, 1991, pp. 104-112.

[15] D.A. Nagy, "Modal Representation of Geometrically Nonlinear Behavior by the Finite Element Method", Computers \& Structures, Vol. 10, 1979, pp. 683-688.

[16] A.K. Noor, "Recent Advances in Reduction Methods for Nonlinear Problems", Computers 8 Structures, Vol. 13, 1981, pp.31-44.

[17] D. S. Park, D. M. Ladd and E. W. Hendricks, "Feedback control of Kármán vortex shedding", Symposium on Active Control of Noise and Vibration, 19-ASME Winter Annual Meeting, Anaheim, CA, 1992.

[18] J.S.Peterson, "The reduced basis method for incompressible viscous flow calculations", SIAM J.Scientific Computing, Vol. 10, 1989, pp. 777-786.

[19] S.W. Roos and J.T. Kegelman, "Control of coherent structures in reattaching laminar and turbulent shear layers", AIA A J., Vol. 24, 1986, pp. 1956-1963.

[20] K. Roussopoulos, "Feedback control of vortex shedding at low Reynolds numbers", Journal of Fluid Mechanics, Vol. 248, 1993, pp. 267-296.

[21] R.L. Sani and P.M. Gresho, "Resume and remarks on the open boundary condition minisymposium", International Journal of Numerical Methods in Fluids", Vol. 18, 1994, pp. $983-1008$.

[22] S. S. Sritharan, "An optimal control problem in exterior hydrodynamics", Proc. of the Royal Society of Edinburgh, Vol. 121 A, 1992, pp. 5-32. 
[23] S. S. Sritharan, Y.-R. Ou, D. S. Park, D. M. Ladd and E. W. Hendricks, "Optimal control of viscous flow past a cylinder: mathematical theory, computation and experiment-Part I-II", Actual Problems of Aviation and Aerospace Systems, Russian-American Scientific Journal, Vol. 1, 1996, pp. 5-156; Vol. 2, 1996, pp. 7-18.

[24] R. Temam, Navier-Stokes Equations and Nonlinear Functional Analysis, SIAM, Philadelphia, PA, 1983. 
TABLE 1. Simulation data for reduced order model: Example I.

\begin{tabular}{lccccccccccc}
\hline \hline & $\mathbf{u}_{1}$ & $\mathbf{u}_{2}$ & $\mathbf{u}_{3}$ & $\mathbf{u}_{4}$ & $\mathbf{u}_{5}$ & $\mathbf{u}_{6}$ & $\mathbf{u}_{7}$ & $\mathbf{u}_{8}$ & $\mathbf{u}_{9}$ & $\mathbf{u}_{10}$ & $\mathbf{u}_{11}$ \\
\hline Velocity on $\Gamma_{t}$ & 1 & 1 & 1 & 1 & 1 & 1 & 1 & 1 & 1 & 1 & 0 \\
Velocity on $\Gamma_{c}[9$ Elements] & 0 & .5 & 1 & 1.5 & -.25 & -.5 & -.75 & -1 & -1.5 & -1.75 & 1 \\
Velocity on $\Gamma_{c}[6$ Elements] & 0 & .5 & 1 & 1.5 & -.25 & -.5 & - & -1 & - & - & 1 \\
Velocity on $\Gamma_{c}[4$ Elements] & 0 & .5 & 1 & - & - & -.5 & - & -1 & - & - & 1 \\
\hline \hline
\end{tabular}

TABLE 2. Simulation data for reduced order model; Example II, Test I.

\begin{tabular}{lccccccccccc}
\hline \hline & $\mathbf{u}_{1}$ & $\mathbf{u}_{2}$ & $\mathbf{u}_{3}$ & $\mathbf{u}_{4}$ & $\mathbf{u}_{5}$ & $\mathbf{u}_{6}$ & $\mathbf{u}_{7}$ & $\mathbf{u}_{8}$ & $\mathbf{u}_{9}$ & $\mathbf{u}_{10}$ & $\mathbf{u}_{11}$ \\
\hline Velocity on $\Gamma_{c}$ [9 Elements] & 0 & -.05 & -.075 & -.1 & -.125 & -.2 & -.3 & -.4 & -.5 & -.6 & -.7 \\
Velocity on $\Gamma_{c}$ [6 Elements] & 0 & - & - & -.1 & - & -.2 & -.3 & -.4 & -.5 & -.6 & -.7 \\
Velocity on $\Gamma_{c}[4$ Elements] & 0 & - & - & -.1 & - & -.2 & -.3 & -.4 & -.5 & - & - \\
\hline \hline
\end{tabular}

TABLE 3. Simulation data for reduced order model; Example II, Test II.

\begin{tabular}{lccccccccccc}
\hline \hline & $\mathbf{u}_{1}$ & $\mathbf{u}_{2}$ & $\mathbf{u}_{3}$ & $\mathbf{u}_{4}$ & $\mathbf{u}_{5}$ & $\mathbf{u}_{6}$ & $\mathbf{u}_{7}$ & $\mathbf{u}_{8}$ & $\mathbf{u}_{9}$ & $\mathbf{u}_{10}$ & $\mathbf{u}_{11}$ \\
\hline Velocity on $\Gamma_{c}[9$ Elements $]$ & 0 & .025 & .05 & .075 & .1 & .125 & .15 & .175 & .2 & .25 & .3 \\
Velocity on $\Gamma_{c}[6$ Elements $]$ & 0 & - & .05 & - & .1 & - & .15 & .175 & .2 & .25 & .3 \\
Velocity on $\Gamma_{c}[4$ Elements $]$ & 0 & - & - & - & .1 & - & .15 & - & .2 & .25 & .3 \\
\hline \hline
\end{tabular}




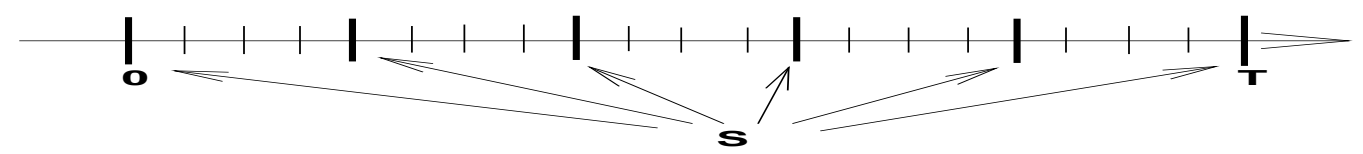

FIG. 1. Illustration of sample selection ("snapshots") for reduced order methods.

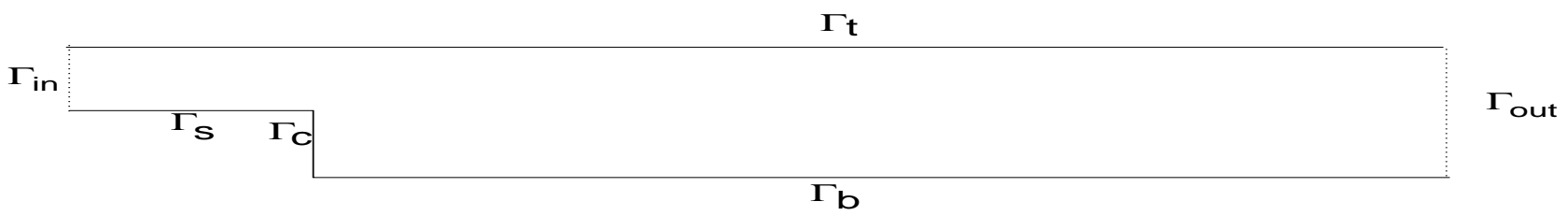

FIG. 2. Computational domain for the backward-facing step channel problem 


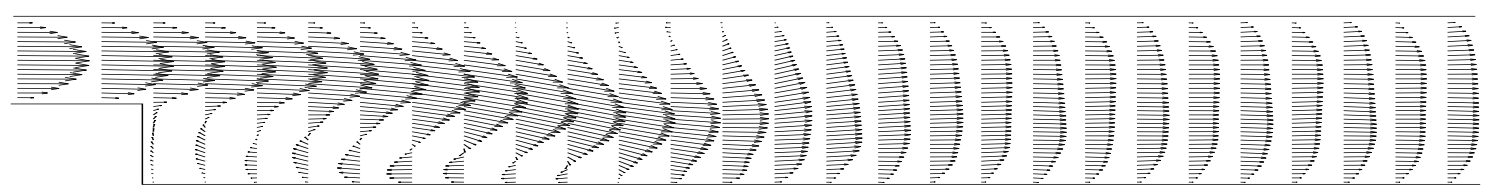

FIG. 3. Full order solution at $t=10$ and for $R e=1000$

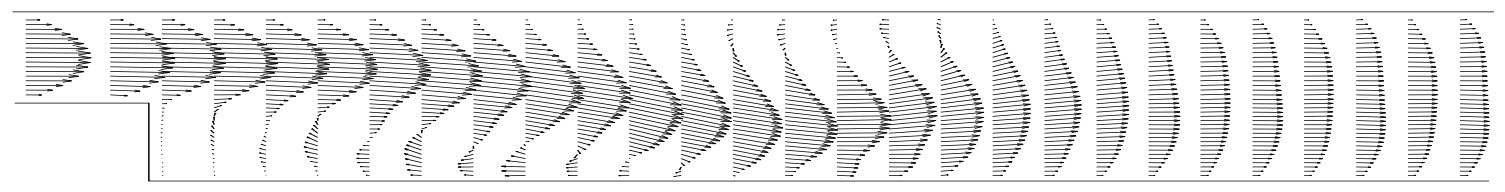

FIG. 4. Reduced order solution at $t=10$ and for $R e=1000$ 


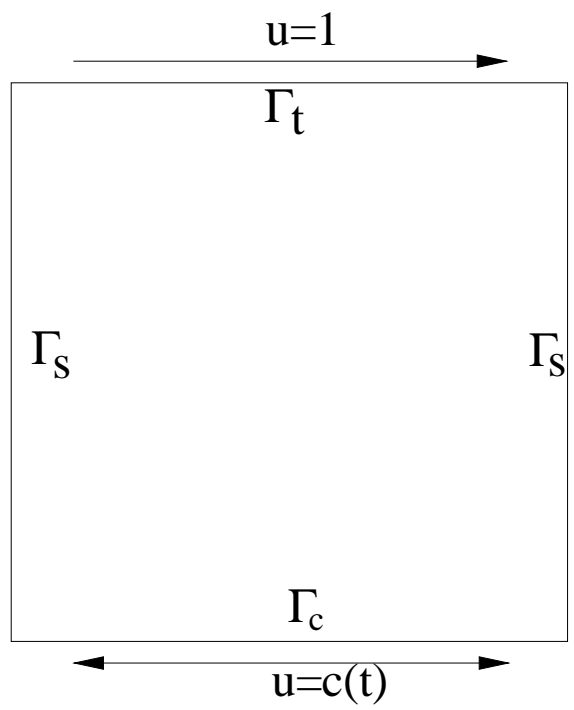

FIG. 5. Computational domain for the driven cavity problem
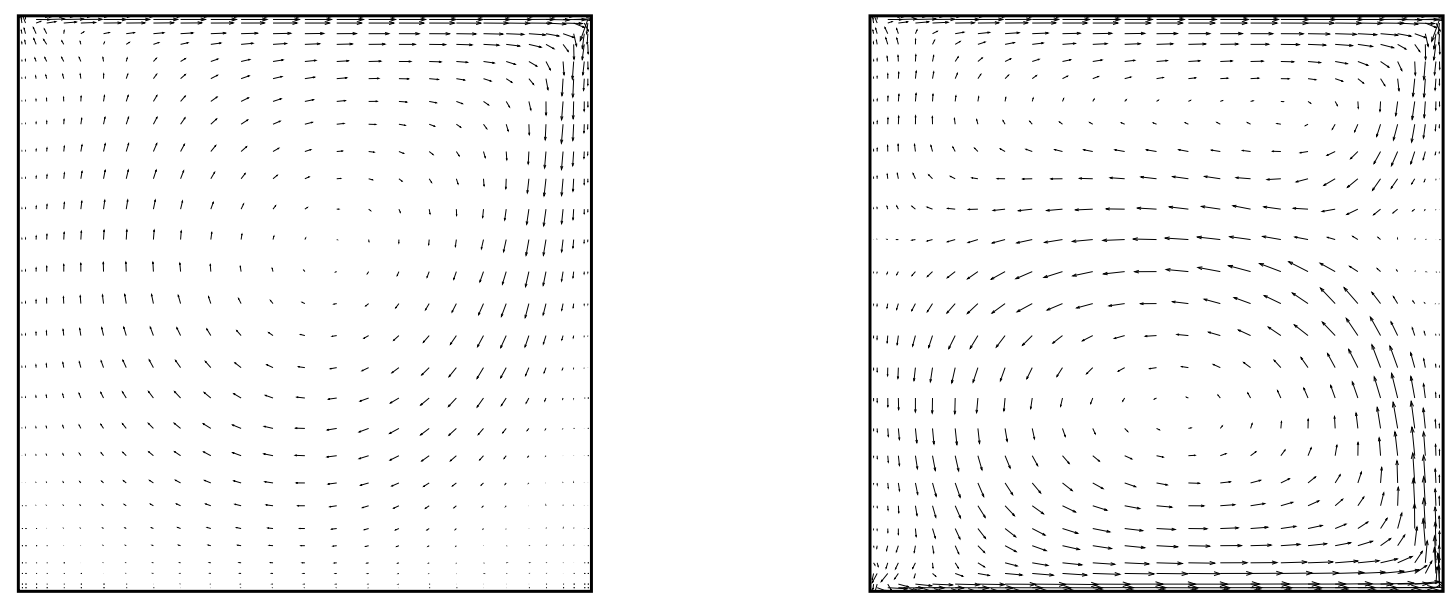

FIG. 6. Driven cavity flow field for Re $=\mathbf{5 0 0}$

FIG. 7. Desired flow field $\mathbf{u}_{d}$ 

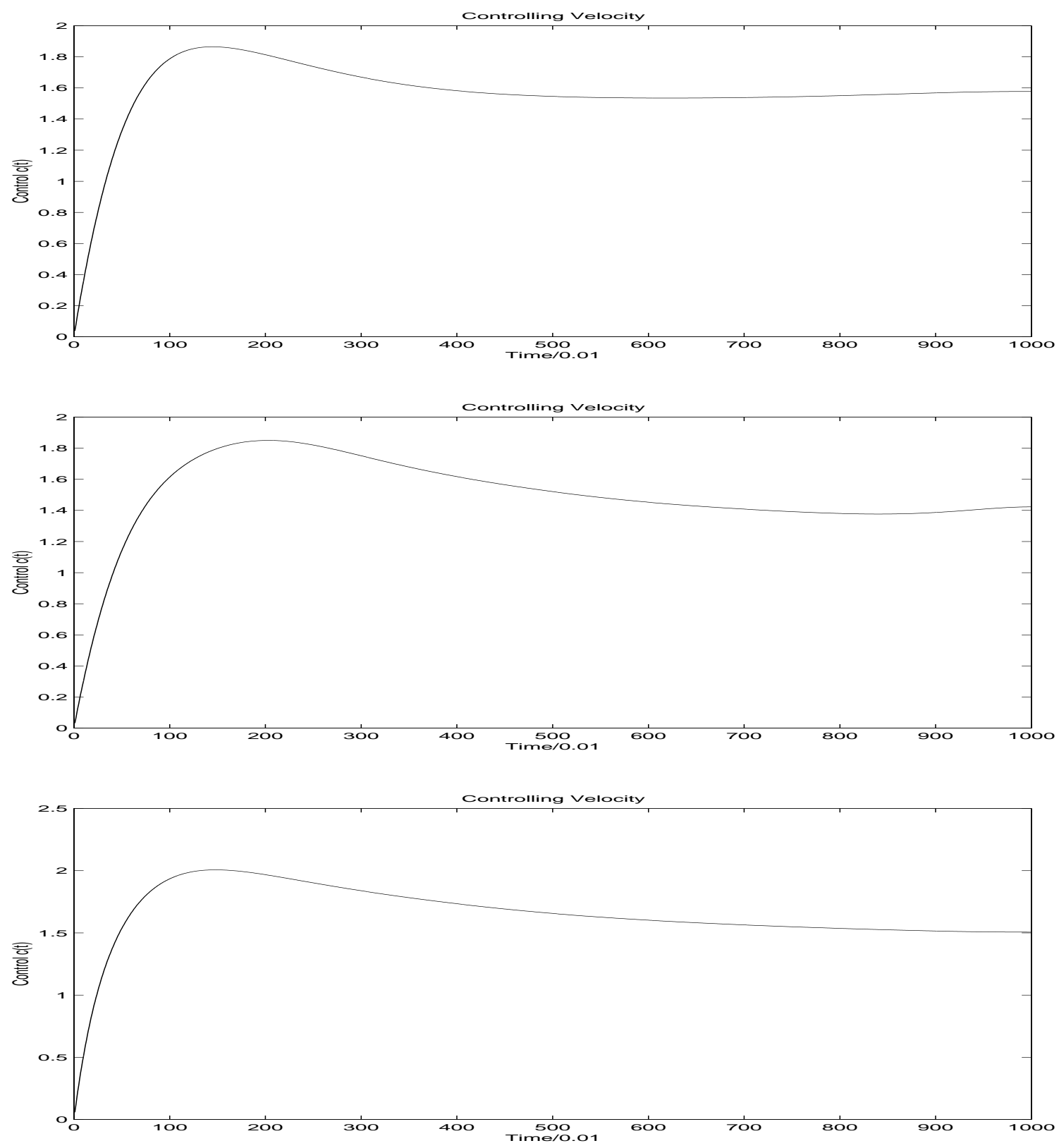

FIG. 8. Optimal controls using 4,6 and 9 RBM basis elements; Example I 


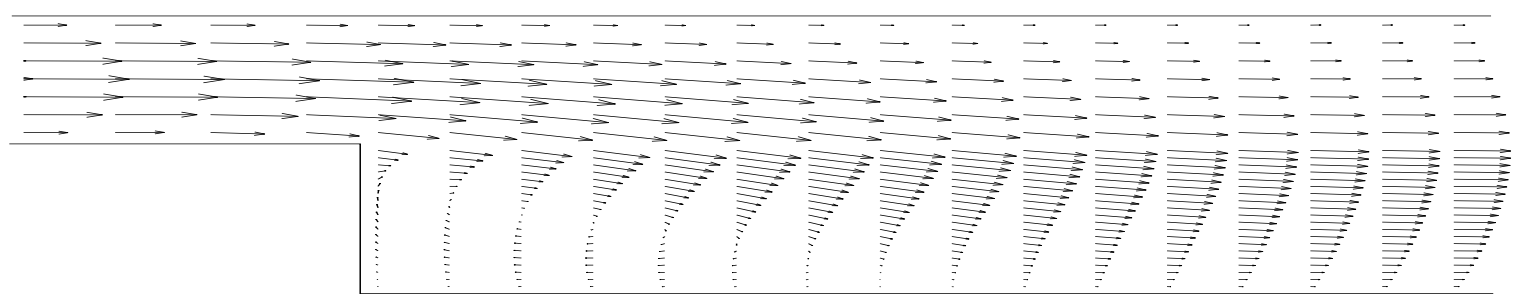

FIG. 9. Uncontrolled velocity field for $\mathrm{Re}=\mathbf{2 0 0}$

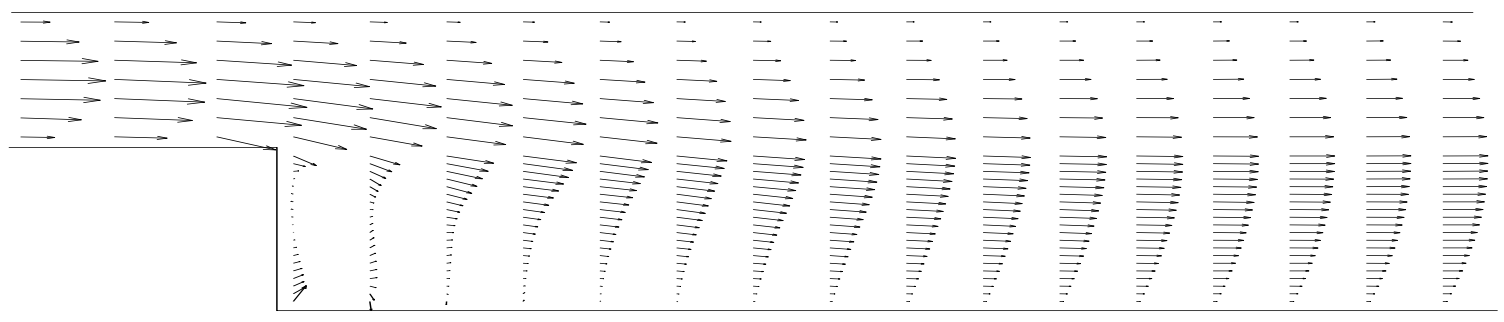

FIG. 10. Channel flow with optimal control input for Re=200 

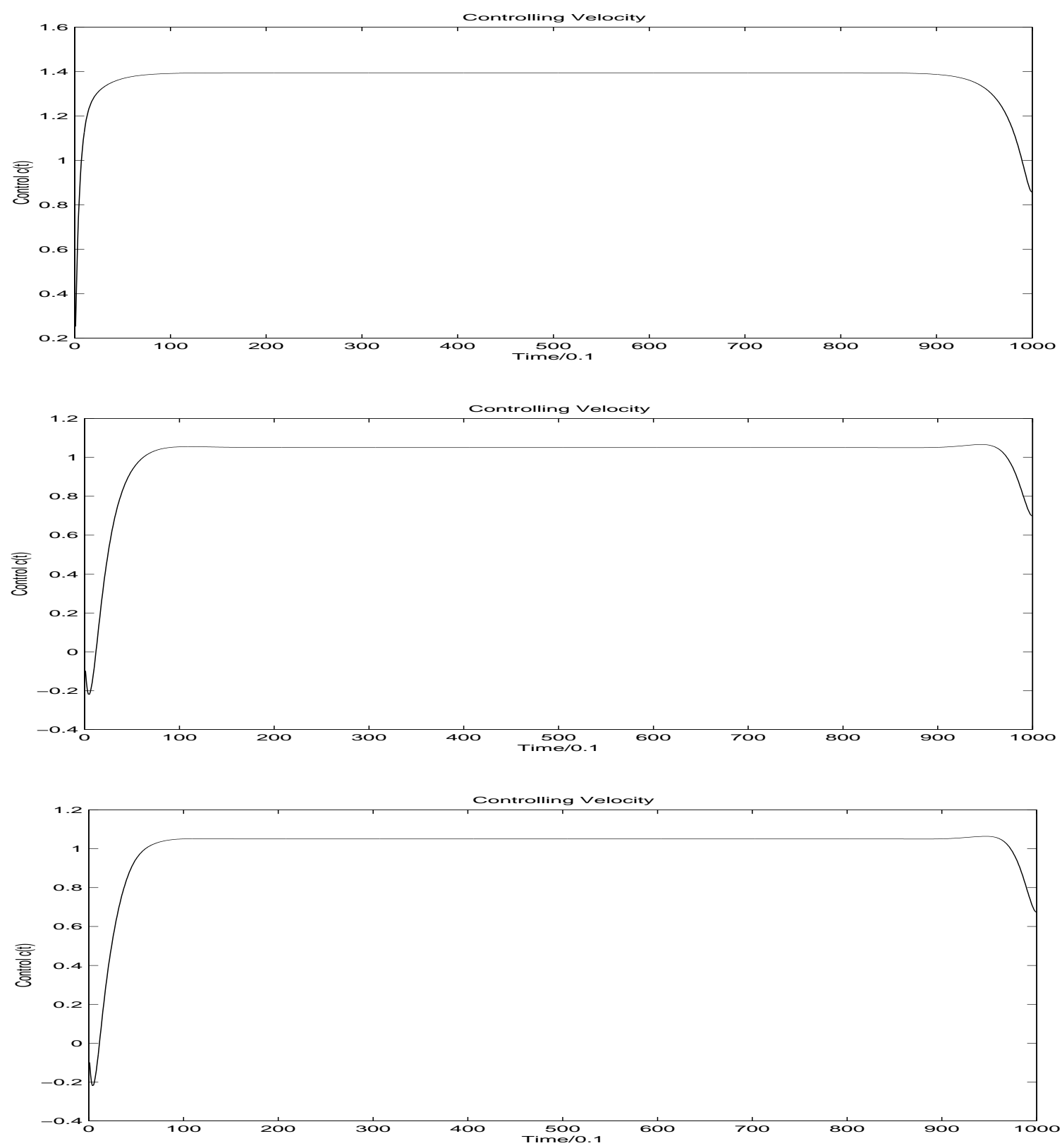

FIG. 11. Optimal controls using 4,6 and 9 RBM basis elements; Example II, Test I 
$\Gamma_{\mathbf{t}}$

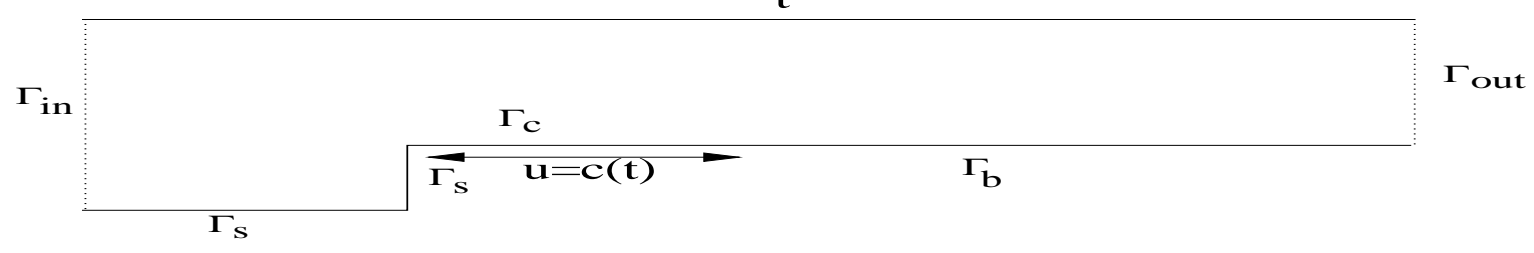

FIG. 12. Computational domain for the forward-facing step channel problem

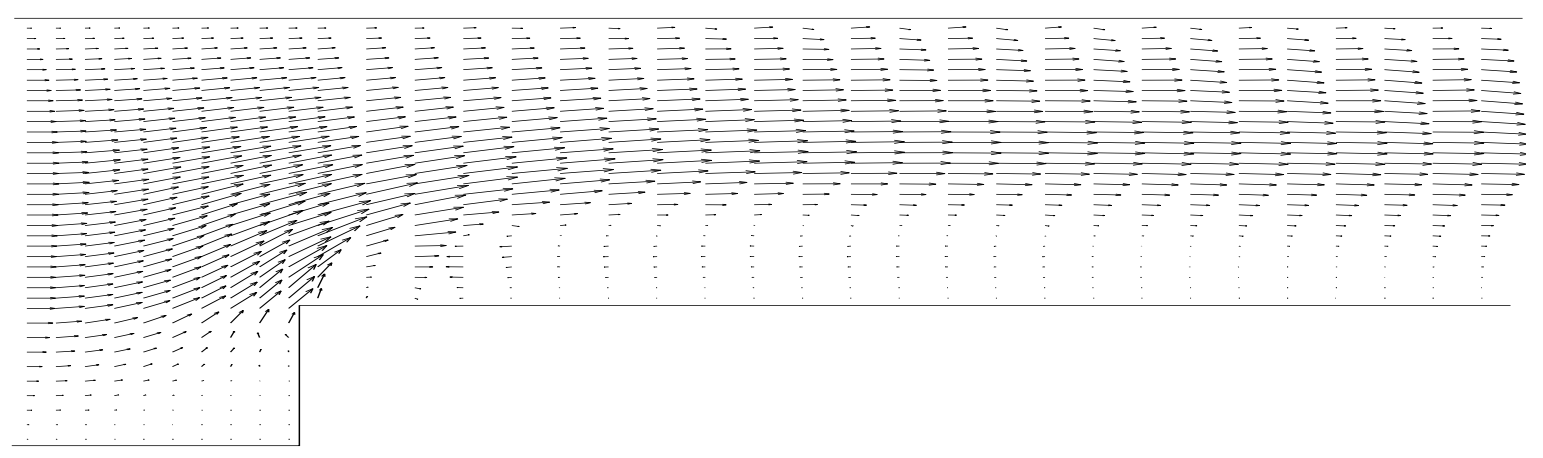

FIG. 13. Uncontrolled velocity field for $R e=1000$ 


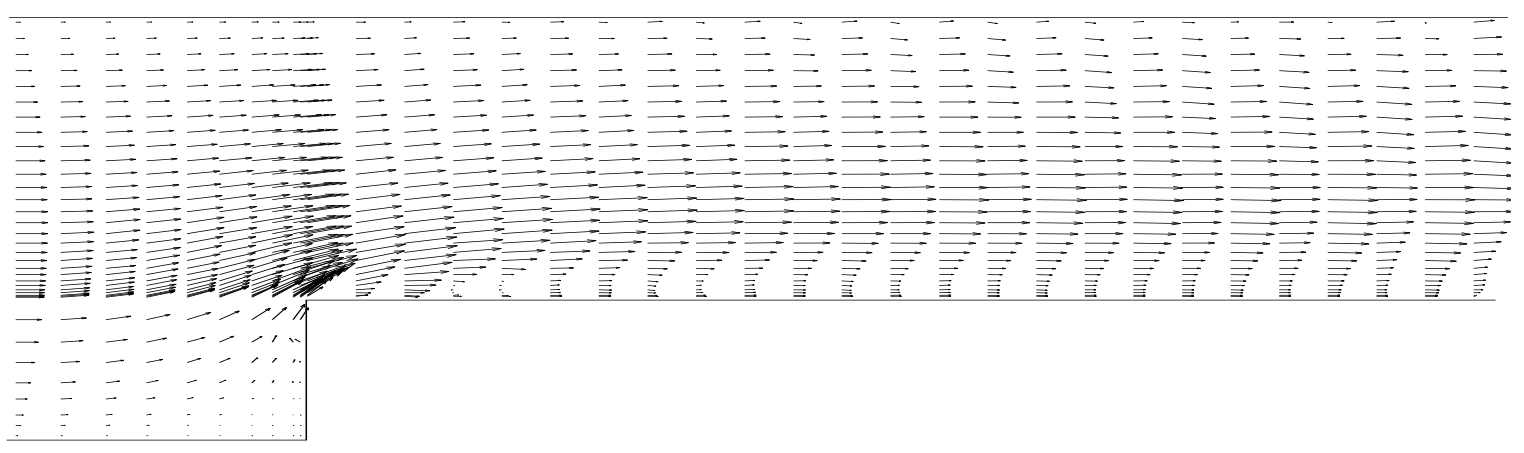

FIG. 14. Channel flow with optimal control input for Re=1000
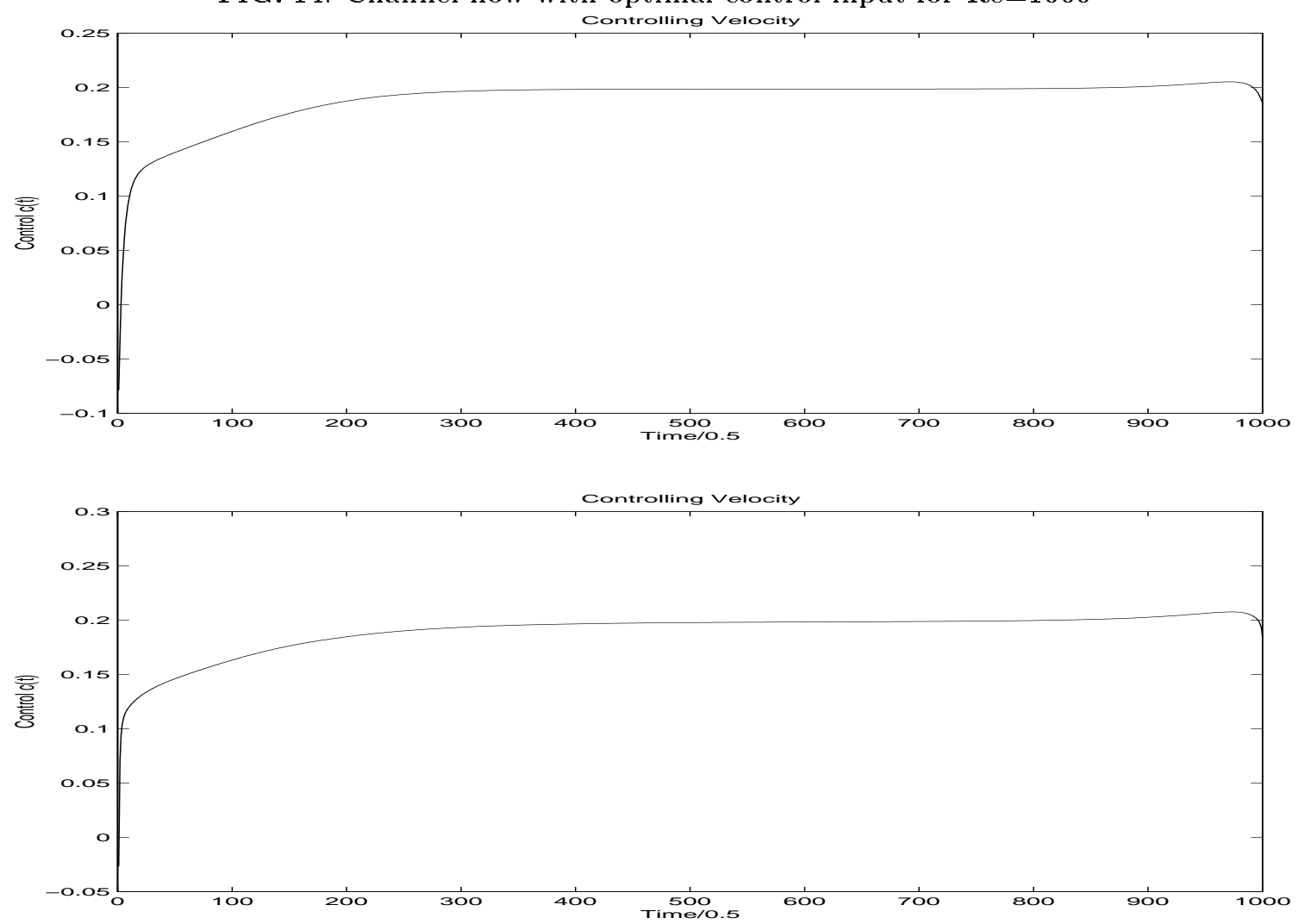

FIG. 15. Optimal controls using 4 and 6 RBM basis elements; Example II. Test II 